\title{
A New Priority Based Call Scheduling Technique in Mobile Networks
}

\author{
Parag Kumar Guha Thakurta
}

\begin{abstract}
A technique for priority driven call scheduling for mobile networks is proposed in this paper. Some priority factors related to call acceptance and call rejection have been identified. A selection criterion for finding the best possible path among the different alternatives obtained through the proposed method has been discussed. Experimental results show the advantages of the proposed technique over existing ones.
\end{abstract}

Index Terms-Priority factors, call scheduling, call acceptance ratio $(\mathrm{CAR})$, routing.

\section{INTRODUCTION}

The explosive growth in the use of mobile devices coupled with users' requirements for real-time applications face a new challenge in the design of scheduling and routing protocols for mobile networks. In particular, it is important that both protocols incorporate the Quality of Service (QoS) metrics for their respective job. The standard cellular model [1] is used to execute these jobs. Users in various cells (different areas of a cellular layout for mobile networks) would communicate through the Base Stations (BSs) and each cell has one BS. Further, these BSs would communicate with each other through a mobile switching centre (MSC).

Under this circumstance, the call scheduling algorithms for mobile networks have several dimensions in terms of the assumptions made, the input space considered and the solution space generated. The three step observations are summarized for such algorithms in [2] as - (a) classifying the algorithms based on the parameters like problem statement, problem goal, type of inputs and solution technique, (b) these algorithms are described with traffic (includes voice, text, video etc.) for application areas such as multi-hop wireless network etc. and (c) finally the algorithms are compared based on classification framework. Based on this review, several existing work are discussed next to address various issues involved with QoS-aware call scheduling techniques.

One of the scheduling algorithms in [3] for multi-hop network was designed to maximize the throughput by reducing call dropping with the application of several enhanced algorithms. A study on the cooperation among the adjacent base stations (BSs) for scheduling in cellular network is discussed in [4]. It increases multi user diversity with the reduction of interference among BSs. Another joint routing and scheduling problem employs multiple accesses in [5]. It includes the provisions for updating the dynamic arrival of new call and terminating the existing calls during the

Manuscript received July 14, 2014; revised December 20, 2014.

Parag Kumar Guha Thakurta is with the National Institute of Technology, Durgapur, India (e-mail: parag.nitdgp@ gmail.com). operation of the network. A mobility model for cellular networks is introduced in [6] to establish a correlation between the accepted and rejected calls.

The Schedule-based Greedy Expansion (S-Expand) algorithm in [7] constructs a tree structure to maximize the call acceptance rate. In this algorithm, the call routing and scheduling are jointly combined in a single phase to allow more flexibility in accepting future calls. Another, tree based routing strategies described in [8] used different approaches than those in [9] for maximizing the call acceptance rate and the number of transmissions. A priority based scheduling algorithm proposed in [10] used a heuristic approach with improved throughput. This scheduling concept was enhanced further by relating the call price and users' demand [11]. It allows a natural prioritization of the calls inside congested environment.

The main focus of the work in this paper is on the scheduling of calls between different BSs through MSC. The model is represented with a graph $\mathrm{G}$, in which a node denotes either a BS or MSC participating in communication among different cells and, an edge between two nodes represents the link if there is a communication between these two. In order to maximize the performance metrics such as the call acceptance ratio (CAR), a scheduling scheme is developed- subsequently a scheduling tree is also obtained. Eventually, the possible alternative paths are identified with respect to the number of hops (intermediate nodes) from the scheduling tree. The problem leads to the determination of some criterion to find the best path amongst all those. The proposed model achieves a major gain over the existing ones - this will be apparent from the discussions in subsequent sections of the paper.

The rest of the paper is organized in the following sections. The system model is described in Section II. The problem address in this paper is described in Section III. Next, the proposed model is presented in Section IV. In Section V, the performance of the model is evaluated with the help of simulation studies. Finally, a conclusion is drawn with a short discussion on advantages of this proposed model in Section VI.

\section{SYSTEM MODEL}

The problem addressed by this work uses the hexagonal cellular layout of mobile networks shown in Fig. 1. Each cell is represented as $C_{r j}$, where ' $r$ ' denotes the radial distance and ' $j$ ' is the sequence from 1 to $\mathrm{n}$ in that particular radius $(r)$. Let us assume that the MSC in in Fig. 1 covers an angle $\theta$ with its axes.

The structure in Fig. 1 can also be represented with a graph structure $[G=(n, e)]$, where, $n$ denotes the set of vertices 
(nodes) and $e$ denotes the set of edges. Here, a node $n_{i}$ represents BSs participated in communication in different cells $\left(C_{r j}\right)$. A directed edge $\left\{C_{a b} \rightarrow C_{e f} \mid C_{a b}, C_{e f} \in n\right\}$ is obtained if it satisfies the following priority factor imposed on the system.

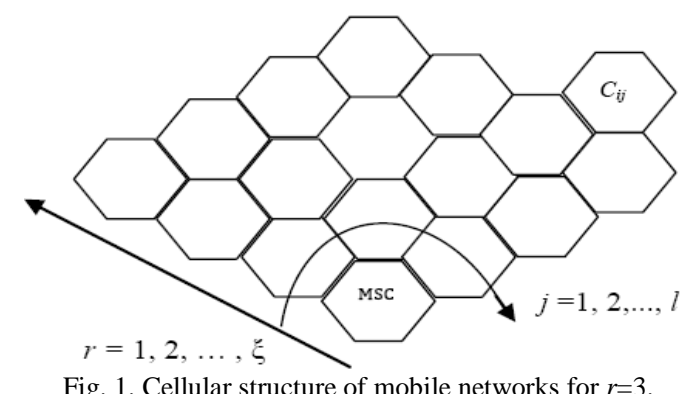

Fig. 1. Cellular structure of mobile networks for $r=3$.

\section{A. Priority Factors}

The cells belonging to a certain value of $r$, that are not in the same column with respect to the cells of lower radius, would become high priority cells for reducing congestion and improving routing sequence. For example, if MSC wants to accept the calls from the cells (BSs) of $r=2$ through $C_{12}$, then it selects $C_{21}$ and $C_{25}$ as high priority cells. The cell numbers $C_{22}$ $C_{23}$ and $C_{24}$ are in the same column of $C_{12}$. So, the edges are constructed as $C_{12} \rightarrow C_{21}$ and $C_{12} \rightarrow C_{25}$. The other priority factors considered are radial distance $r$ and propagation delay. It is known that the propagation delay is proportional to the distance between MSC and the BS. So, the nearest BS from MSC would be assigned high priority. Identification of these parameters provides necessary support to differentiate incoming calls.

\section{B. Performance Metric}

Based on the discussion on priority factors, following two QoS parameters are introduced here to demonstrate the effectiveness of the proposed model.

\section{1) Call acceptance ratio (CAR)}

The call acceptance ratio (CAR) is defined as $(\lambda / \mu)$, where $\lambda$ is the number of BSs in radius $(r+1)$, selected on the basis of the priority values, with respect to any $\mathrm{BS}$ in radius $r$; $\mu$ is the total number of BSs present in radius $(r+1)$.

The reason for using the call acceptance ratio rather than the call acceptance rate [8] is that - the selection of nodes is dependent on the interrelation between two successive radii like $r$ and $(r+1)$ respectively; earlier the call acceptance rate was computed with respect to the entire system and not on such detailed scheduling of nodes.

Lemma: The effect of priority factors is identical irrespective of the layout.

Proof: The values of angle $\theta$ can be $2 \pi, \pi, 2 \pi / 3$ and $\pi / 3$ due to the symmetric nature of the hexagonal structure. The CAR values would increase proportionally with the increment of the value of $r$ for the range $0<=\theta<=2 \pi / 3$. The non uniformity of CAR values can be seen for other possible ranges of $\theta$ like $\theta<=\pi / 3$ and $\theta>2 \pi / 3$. Hence, the total number of cells in a specific radius level $r$ would be $(2 r+1)$ with $\theta \in[0,2 \pi / 3]$. So, the cellular structure is symmetric in all axes and therefore the priority factors would be same in any direction irrespective of the axes.

\section{2) Utilization Factor $(U F)$}

The Utilization Factor (UF) is defined as a product between the call acceptance ratio (CAR) and the time period $t_{p}$ where $t_{p}$ denotes the different periods of time during a day according to the requirement of mobile usage.

\section{PROBLEM STATEMENT}

When different nodes communicate with each other through MSC within the transmission range $(\forall i \in r)$, the priority factors play an important role with respect to call scheduling. The objective of the problem is to find a scheduling tree $(T)$ from the given graph $G$ in order to maximize CAR- this maximization of CAR leads to improved performance for the proposed model with respect to call acceptance. In addition, the alternative paths $\left(P_{i}\right)$ are found from $T$ and then, the best one among these alternatives is determined.

\section{PROPOSED MODEL}

The model proposed in this work is executed in two phases. The scheduling algorithm is executed first followed by the selection of the best path among the alternatives obtained from $T$.

\section{A. Call Scheduling Algorithm}

The algorithm generates a scheduling tree of the calls originated from different BSs satisfying the priority factors. The MSC accepts calls from any BS in $r=1$ within the angle $\Theta$ as a base condition. In the following $\lambda$ and $\partial$ represents total number of cells in radius distance $r$ and $(r+1)$ respectively; $\mu$ and $\mu 1$ are $\lceil\lambda / 2\rceil$ and $\lceil\partial / 2\rceil$ respectively being used to provide the middle value as the total number of cells in a specific radius level $r$ would be an odd number; $C_{r_{c}}$ gives cell $C$ in radius $r$ with the serial number $c$. Hence, the value of $c$ has been compared with the different values of $\mu$. The initial value of both $\lambda$ and $\partial$ are taken to be $3+2(r-1)$.

\section{Algorithm: Call scheduling}

Input: Graph (G)

Output: Scheduling tree $(\mathrm{T})$

procedure $\operatorname{CSA}(\mathrm{G}, \mathrm{T})$

begin

$$
\begin{aligned}
& r \leftarrow 1 / / /^{*} \text { radius is initialized } * / / \\
& c \leftarrow 1 / / * \text { serial number is initialize } \\
& \text { for } r=1 \text { to } n / / /^{*} n \text { is the maximum } \\
& \text { loop } \\
& \text { parent_node } \leftarrow C_{r c} \\
& \lambda=2 r+1 \\
& \partial=2 r+3 \\
& \mu=\lceil\lambda / 2\rceil \\
& \mu 1=\lceil\partial / 2\rceil \\
& r^{*}=r+1 \\
& c^{*}=c+1 \\
& c^{* *}=c+2 \\
& \mu 1^{*}=\mu 1+1 \\
& \mu 1=\mu 1-1
\end{aligned}
$$$$
c \leftarrow 1 / / * \text { serial number is initialized } * / /
$$$$
\text { for } r=1 \text { to } n / / *_{n} \text { is the maximum radius } * / /
$$

for each cell $C_{\left(r^{*} c^{\prime}\right)} / /{ }^{*} c^{\prime} \in[1, \partial]$ for next higher radius 
level*//

loop

if $(r>0)$

if $(c<\mu)$

if $\left(C_{r^{*} c}, €\left\{C_{r^{*} c}, C_{r^{*} c^{*}}, C_{r^{*} \mu 1^{*}}\right\}\right)$

reject $C_{r *}$,

end if

else if $(c=\mu)$

if $\left(C_{r^{*} c}, €\left\{C_{r^{*} \mu 1}, C_{r^{*} \mu 1}, C_{r^{*} \mu 1 *}\right\}\right)$

reject $C_{r^{*}} c$

end if

else

if $\left(C_{r^{*} c^{\prime}}, \mathcal{\in}\left\{C_{r^{*} \mu 1}, C_{r^{*} c^{*}}, C_{r^{*} c^{* *}}\right\}\right)$

reject $C_{r * c}$

end if

end if

end if

end if

add $C_{r^{*} c^{\prime} \text { as }}$ a child to the parent node //*adding

consequent children*//

end loop

end loop

$C \leftarrow C^{\prime} / / *^{\text {go }}$ to the next radius level $* / /$

end

Example: The scheduling algorithm is explained here for $r$ $=2$. The MSC can include any cell $C_{11}, C_{12}$ or $C_{13}$ of $r=1$ according to base condition of the algorithm. Consider, the cell number $C_{11}$ for $r=1$ and $c=1$. Here the values of both $\lambda$ and $\partial$ are 3 and those of $\mu$ and $\mu 1$ are 2. As, $c<\mu$, the cell numbers $C_{21}, C_{22}$ and $C_{24}$ are rejected by MSC. Similarly, if MSC could accept the call requests through $C_{12}$, then the cell numbers $C_{22}, C_{23}$ and $C_{24}$ are not accepted. Subsequently, the cell numbers $C_{22}, C_{24}$ and $C_{25}$ are excluded if MSC could accept the call requests through $C_{13}$. Fig. 2 shows the tree structure obtained from the algorithm.

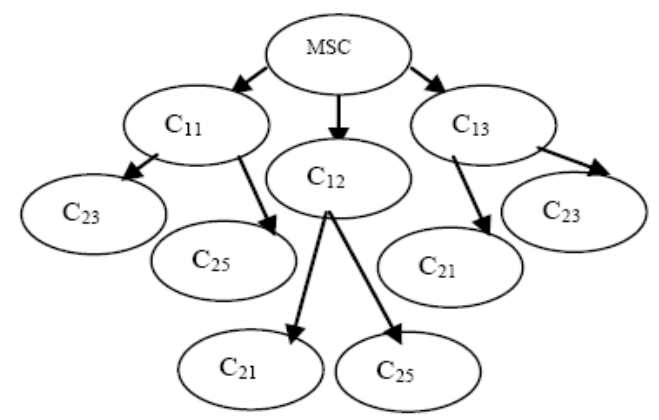

Fig. 2. $T$ with $r=2$.

Time Complexity: The height of the tree grows as $O\left(\log _{m}\right.$ $n$ ), where $\mathrm{m}$ and $\mathrm{n}$ denote the degree of the node and the total number of nodes of the tree respectively [12]. Obviously, the height of the tree is related to the scheduling of the calls. Further, adjusting the node in the priority level takes an additional $O(m)$ time. Therefore, the total time needed is $O$ $\left(m+\log _{m} n\right)$ for call scheduling.

\section{B. Selection of Best Possible Path}

The scheduling tree thus obtained has been transformed into a binary tree [13] for the generation of path sequence using the prefix code notation. Thereafter, it would facilitate identification of path sequences corresponding to each BS of call requesting cell with respect to MSC. The path is used to track the signal following the route between source and destination. The use of prefix code notation determines the path consisting of sequence of 0 and 1 . For example, the alternative paths obtained for $\mathrm{C}_{37}$ to reach MSC are 000111 or 010011 or 0110011.

Now it is necessary to find the possible solution among these alternatives to improve routing performance. The possible selection criteria are described in the following instances.

1) $\operatorname{Min}\left(|L| \epsilon S_{0,1}\right), \forall L_{i} \neq L_{j}$, where $L_{i}$ and $L_{j}$ represent the code lengths of different paths and $\left(S_{0,1}\right)$ denotes the binary path sequence.

2) $\operatorname{Max}(R)=\min \left\{L \in S_{0,1}\right\}, \forall L_{i}=L_{j}$, where $L_{i}$ and $L_{j}$ represent the code lengths of different paths and $\left(S_{0,1}\right)$ denotes the binary path sequence. Here, $R$ counts the number of $1 \mathrm{~s}$ which can be computed from an expression like $\left(n_{1} \oplus n_{2}\right)+\left(n_{2} \oplus n_{3}\right)+\ldots \ldots \ldots \ldots+\left(n_{k-2} \oplus n_{k-1}\right)+$ $\left(n_{k-1} \oplus n_{k}\right)$, where $L$ denotes a n-bit $S_{0,1}$.

Example: The selection criteria find 00 as the best one among the alternatives for BS in cell number $\mathrm{C}_{23}$. Similarly, the $\mathrm{BS}$ in cell numbers $\mathrm{C}_{36}$ has more than one minimum code length path sequences like 00011 and 01001 Here, the best possible path has been found to be 01001 [14], [15].

\section{Simulation Studies}

The simulation of the proposed model has been carried out using MATLAB 7.6.0, C\# and .Net 4.0 on a server with two Intel Xenon processor $(2.33 \mathrm{GHz})$ and $16 \mathrm{~GB}$ memory.

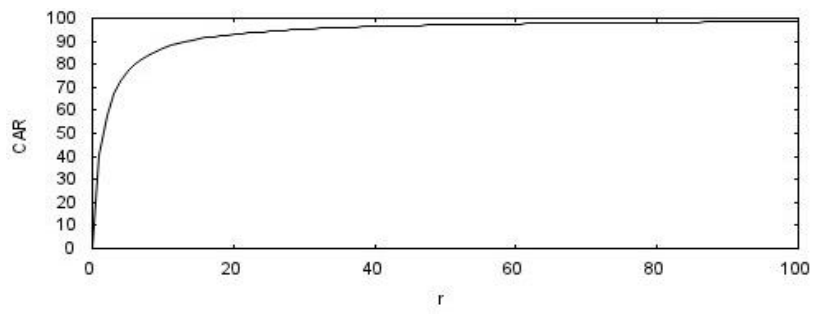

Fig. 3. Nature of CAR.

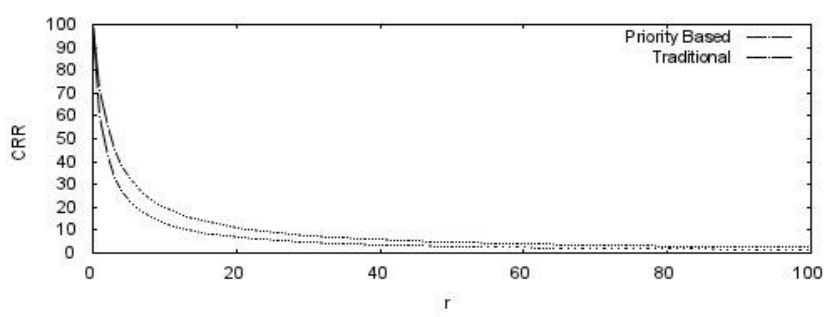

Fig. 4. Improvement in terms of CRR.

The model proposed here shows (in Fig. 3) the nature of $C A R$ with different values of $r$. Here, the range of the values for $r$ is taken as $\{0: 100\}$ and subsequent $\operatorname{CAR}\left\{(\lambda / \mu) \forall C_{r j}, j \in\right.$ l) are observed. In Fig. 4, an improvement over the works presented in [7], in terms of the call rejection ratio $(C R R)$ which is the complement of $C A R$ is shown with similar rage of $r$. Next, Fig. 5 shows that the performance of $C A R$ follows the S-curve when compared with some standard types and it meets the requirement of traffic forecasting for mobile networks [16]. The summary of the parameters used is shown in Table I. Here, R. Square denotes root square value for each 
standard equation. The other columns like b1, b2, b3 denote corresponding coefficients in equations.

y

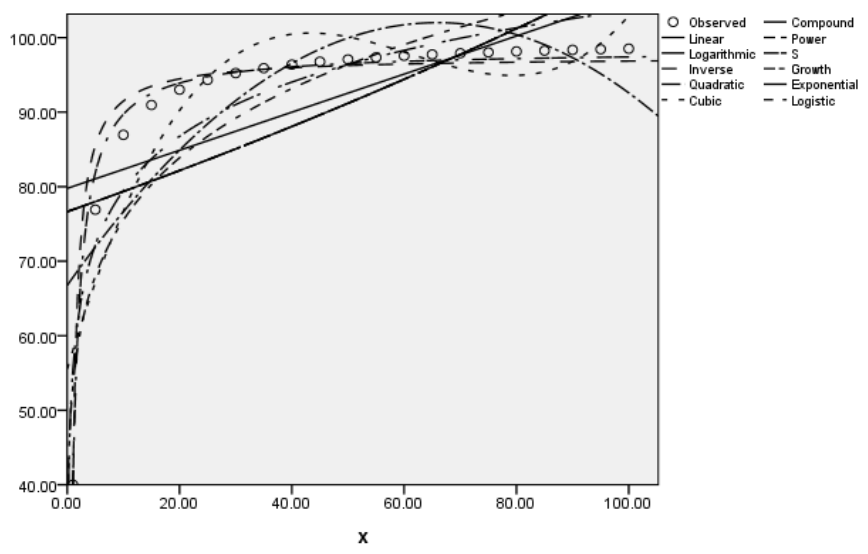

Fig. 5. Curve fitting for CAR.

Generally, the number of calls admitted during a day follows a Poisson distribution [6]. The proposed model achieves better system utilization (in terms of the $U F$ ) as shown in Fig. 6, over the existing approaches (like traditional routing algorithms, alternative based approaches in [6], [7]). It is important to mention that the improved $U F$ (in $\mathrm{Y}$-axis) would be noticeable during peak hours of a day (shown in $\mathrm{X}$-axis).

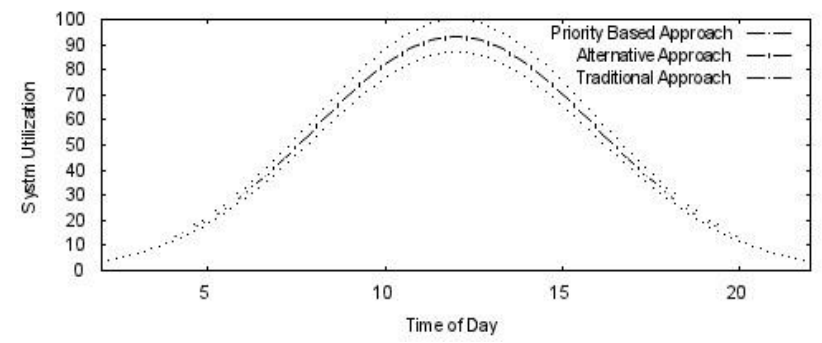

Fig. 6. Comparative study between different scheduling approaches.

TABLE I: MODEl SumMARY (THE DEPENDENT VARIABLE IS CAR AND THE INDEPENDENT VARIABLE IS $R$ )

\begin{tabular}{|c|c|c|c|c|c|}
\hline \multirow{2}{*}{ Equation } & \multicolumn{5}{|c|}{ Model Summary } \\
\cline { 2 - 6 } & R Square & Constant & b1 & b2 & b3 \\
\hline Linear & .366 & 79.772 & .256 & & \\
\hline Logarithmic & .833 & 55.277 & 10.507 & & \\
\hline Inverse & .962 & 97.451 & -59.675 & & \\
\hline Quadratic & .630 & 66.753 & 1.070 & -.008 & \\
\hline Cubic & .796 & 55.527 & 2.546 & -.045 & .000 \\
\hline Compound & .297 & 76.650 & 1.003 & & \\
\hline Power & .760 & 53.256 & .152 & & \\
\hline S & .992 & 4.588 & -.915 & & \\
\hline Growth & .297 & 4.339 & .003 & & \\
\hline Exponential & .297 & 76.650 & .003 & & \\
\hline Logistic & .297 & .013 & .997 & & \\
\hline
\end{tabular}

\section{CONCLUSIONS}

This paper presents a priority-driven call scheduling approach for mobile networks with logarithmic execution time. Most of the authors resolved the scheduling problem using the broad concept of priority- however they did not integrate the idea of priority factors in designing the scheduling scheme. In this paper we therefore integrate these factors for call scheduling in a more comprehensive manner. Thus, an improvement is obtained over existing approaches. In this paper, the importance of CAR has been established. Further, possible paths for call scheduling have been determined and a method for selection of the best path from the set of paths thus obtained has also been suggested. Currently, work is being undertaken to introduce a robust path selection technique beyond the one presented here.

\section{REFERENCES}

[1] P. K. G. Thakurta and S. Bandyopadhyay, "A new dynamic pricing scheme with priority based tree generation and scheduling for mobile networks," in Proc. IEEE Advanced Computing Conference, March 2009.

[2] V. Gabale, B. Raman, P. Dutta, and Sh. Kalyanraman, "A classification framework for scheduling algorithms in wireless mesh networks," IEEE Communications Surveys \& Tutorials, January 2012

[3] M. K. R. Al-juaifari and B. P. Rani, "Engineering wireless mesh networks: Joint scheduling, routing, power control and rate adaptation networks with bottleneck algorithm," International Journal of Scientific \& Engineering Research, vol. 3, issue 9, September 2012.

[4] M. Dianati, X. Shen, and K. Naik, "Cooperative fair scheduling for the downlink of CDMA cellular networks," IEEE Transactions on Vehicular Technology, vol. 56, pp. 1749-1760, July 2007.

[5] A.-A. Mahmood and E. S. Elmallah, "An algorithm for incremental joint routing and scheduling in wireless mesh networks," IEEE WCNC, 2010, pp. 1-6.

[6] S. Yaipairoj and F. C. Harmantzis, "Dynamic pricing with 'Alternatives' for mobile networks," in Proc. WCNC. 2004, IEEE, vol. 2, pp. 671-676.

[7] H. S. Chiu and K. L. Yeung, "A joint routing and scheduling algorithm for efficient broadcast in wireless mesh networks," in Proc. IEEE WCNC, 2010.

[8] H. S. Chiu and K. L. Yeung, "Maximizing multicast call acceptance rate in multi-channel multi-interference wireless mesh network," IEEE Transactions on Wireless Communications, vol. 9, no. 8, pp. 26222631, August 2010.

[9] P. K. G. Thakurta, R. Poddar, and S. Bandyopadhyay, "A new approach on co-ordinate based routing protocol for mobile networks," in Proc. IEEE Advanced Computing Conference, Patiala, Punjab, India, February 2010, pp. 177-180

[10] M. Dianati, X. Shen, and K. Naik, "Cooperative fair scheduling for the downlink of CDMA cellular networks," IEEE Transactions on Vehicular Technology, vol. 56, no. 4, pp. 1749-1760, July 2007.

[11] S. Yaipairoj and F. C. Harmantzis, "Priority telephony system with pricing alternatives," in Proc. ICT, Fortaleza, Brazil, August 2004, LNCS 3124, pp. 183-191.

[12] H. Lu and S. Sahani, "B-tree router table design," IEEE Transaction on Computers, vol. 54, issue 7, pp. 813-824, July 2005.

[13] D. E. Knuth, "The art of computer programming," Fundamental Algorithms, Addison-Wesley, Third Edition, 2007, vol. 1, pp. 354-355.

[14] S. Ramprasad, N. R. Shanbhag, and I. N. Hajj, "Signal coding for low power: fundamental limits and practical realizations," IEEE Transactions on Circuits and Systems II: Analog and Digital Signal Processing, vol. 46, issue 7, pp. 923-929, July, 1999.

[15] H. Chan, P. Fan, and Z. G. Cao, "A utility-based network selection scheme for multiple services in heterogeneous networks," in Proc. International Conference on Wireless Networks, Communications and Mobile Computing, 13-16 June, 2005, pp. 1175-1180.

[16] I. Z. Kovacs, P. Mogensen, B. Christensen, and R. Jarvela, "Mobile broadband traffic forecast modeling for network evolution studies," in Proc. IEEE Vehicular Technology Conference, Budapest, Hungary, May 2011, pp. 1-5.

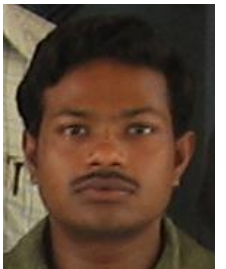

Parag Kumar Guha Thakurta is currently working as an assistant professor in the Department of Computer Science \& Engineering at National Institute of Technology, Durgapur, India. He received M.Tech degree in computer science \& engineering from University of Calcutta. His area of research interest is mobile computing. 